\title{
BOUNDARY VALUE PROBLEMS WITH REGULAR SINGULARITIES AND SINGULAR BOUNDARY CONDITIONS
}

\author{
G. FREILING AND V. YURKO
}

Received 14 May 2004

Singular boundary conditions are formulated for nonselfadjoint Sturm-Liouville operators with singularities and turning points. For boundary value problems with singular boundary conditions, properties of the spectrum are studied and the completeness of the system of root functions is proved.

\section{Introduction}

We consider a class of singular differential equations of the form

$$
-\frac{d}{d t}\left(p_{2}(t) \frac{d z}{d t}\right)+p_{1}(t) z(t)=\lambda p_{0}(t) z(t), \quad t \in(a, b) .
$$

Here $\lambda$ is the spectral parameter, and the complex-valued functions $p_{k}(t)$ have zeros or/and singularities at the endpoints of the interval $(a, b)$. More precisely,

$$
p_{k}(t)=(t-a)^{s_{k 0}}(b-t)^{s_{k 1}} p_{k 0}(t),
$$

where $s_{k m}$ are real numbers, $p_{k 0}(t) \in C^{2}[a, b], p_{00}(t) p_{20}(t) \neq 0, p_{00}(t) / p_{20}(t)>0$ for $t \in$ $[a, b]$. Let $s_{2 m}<s_{0 m}+2, s_{2 m} \leq s_{1 m}+2, m=0,1$, that is, we consider the case of so-called regular singularities. Operators with irregular singularities possess different qualitative properties and require different investigations.

Since the solutions of (1.1) may have singularities at the endpoints of the interval, and since in general the values of the solutions and their derivatives at the endpoints are not defined, an important question is how to introduce singular two-point boundary conditions in the general case under consideration. For some particular cases this problem has been studied in $[4,5,6,15,21,23]$ and other works. For example, in [4] singular boundary conditions were constructed in the case when the endpoints are of limit-circle type.

In this paper, we provide a general method for defining two-point singular boundary conditions in the above-mentioned general case. In Section 2, we construct singular boundary conditions and formulate the corresponding boundary value problems. In Section 3, properties of the spectrum are studied for boundary value problems with 
singular boundary conditions. In Section 4, the completeness of the system of eigen - and associated functions (eaf's) is proved for this class of boundary value problems.

We mention that the approach presented in this paper can serve as a basis for various investigations connected with the spectral theory of Sturm-Liouville equations (and also for higher-order differential equations and systems) with singular boundary conditions. Further topics connected with problems with singular boundary conditions, like, for example, expansion theorems and inverse spectral problems, will be studied elsewhere.

For simplicity, we confine ourselves here to the case when there are no singularities and turning points inside the interval. We note that spectral problems for ordinary differential operators without singularities (or with integrable coefficients) were investigated in many works (see the monographs $[10,12,13,16,17,19]$ and the references given therein). Some aspects of spectral problems for differential equations having singularities and/or turning points with classical boundary conditions at the endpoints were studied among others in $[1,3,7,9,11,14,18,22,24]$, where further references can be found.

\section{Singular boundary conditions}

Denote

$$
\begin{gathered}
r(t)=\frac{p_{0}(t)}{p_{2}(t)}, \quad \chi(t)=\frac{p_{1}(t)}{p_{2}(t)}+\frac{d}{d t}\left(\frac{\dot{p}_{2}(t)}{2 p_{2}(t)}\right)+\left(\frac{\dot{p}_{2}(t)}{2 p_{2}(t)}\right)^{2}, \\
R(t)=(r(t))^{1 / 2}>0, \quad T=\int_{a}^{b} R(\xi) d \xi, \quad s_{m}=s_{0 m}-s_{2 m}, \quad m=0,1 .
\end{gathered}
$$

Then $s_{m}>-2, m=0,1$, and there exist the finite limits

$$
\chi_{0}=\lim _{t \rightarrow a+0}(t-a)^{2} \chi(t), \quad \chi_{1}=\lim _{t \rightarrow b-0}(b-t)^{2} \chi(t) .
$$

Denote

$$
v=\frac{2}{s_{0}+2}\left(\chi_{0}+\frac{1}{4}\right)^{1 / 2}, \quad \gamma=\frac{2}{s_{1}+2}\left(\chi_{1}+\frac{1}{4}\right)^{1 / 2} .
$$

For definiteness, let $\operatorname{Re} v>0, \operatorname{Re} \gamma>0, v, \gamma \notin \mathbb{N}$ (other cases require minor modifications). We transform (1.1) by means of the replacement

$$
x=\int_{a}^{t} R(\xi) d \xi, \quad y(x)=\left(p_{0}(t) p_{2}(t)\right)^{1 / 4} z(t)
$$

to the differential equation

$$
-y^{\prime \prime}(x)+q(x) y(x)=\lambda y(x), \quad x \in(0, T),
$$

where $q(x)=\ddot{r}(t)\left(4 r^{2}(t)\right)^{-1}-5 \dot{r}(t)\left(16 r^{3}(t)\right)^{-1}+\chi(t)(r(t))^{-1}$. The function $q(x)$ is continuous for $x \in(0, T)$, and it has second-order singularities at the endpoints of the interval:

$$
q(x)=\frac{\omega}{x^{2}}+q_{0}(x), \quad x \in\left(0, \frac{T}{2}\right], \quad q(x)=\frac{\omega_{1}}{(T-x)^{2}}+q_{0}(x), \quad x \in\left(\frac{T}{2}, T\right),
$$


where $\omega=v^{2}-1 / 4, \omega_{1}=\gamma^{2}-1 / 4$. We assume that $q_{0}(x) x^{2 \theta}(T-x)^{2 \theta_{1}} \in \mathscr{L}(0, T)$, where $\theta:=1 / 2-\operatorname{Re} v, \theta_{1}:=1 / 2-\operatorname{Re} \gamma$.

First of all, we construct fundamental systems of solutions (FSSs) for (2.5) having power-type behavior near the endpoints of the interval $(0, T)$. Let $\lambda=\rho^{2}, \arg \rho \in$ $(-\pi / 2, \pi / 2]$. Consider the functions

$$
C_{j}(x, \lambda)=x^{\mu_{j}} \sum_{k=0}^{\infty} c_{j k}(\rho x)^{2 k}, \quad j=1,2,
$$

where

$$
\begin{gathered}
\mu_{j}=(-1)^{j} \nu+\frac{1}{2}, \quad c_{10} c_{20}=(2 \nu)^{-1} \\
c_{j k}=(-1)^{k} c_{j 0}\left(\prod_{s=1}^{k}\left(\left(2 s+\mu_{j}\right)\left(2 s+\mu_{j}-1\right)-\omega\right)\right)^{-1} .
\end{gathered}
$$

Here and in the sequel, $z^{\mu}=\exp (\mu(\ln |z|+i \arg z)), \arg z \in(-\pi, \pi]$. It can be easily verified that the functions $C_{j}(x, \lambda), j=1,2$, are solutions of the equation $-y^{\prime \prime}+\omega x^{-2} y=\lambda y$.

Let $S_{j}(x, \lambda), j=1,2$, be solutions of the following integral equations:

$$
S_{j}(x, \lambda)=C_{j}(x, \lambda)+\int_{0}^{x} g(x, t, \lambda)\left(q(t)-\omega t^{-2}\right) S_{j}(t, \lambda) d t, \quad 0<x<T,
$$

where $g(x, t, \lambda)=C_{1}(t, \lambda) C_{2}(x, \lambda)-C_{1}(x, \lambda) C_{2}(t, \lambda)$. The properties of the functions $S_{j}(x, \lambda)$ and of the corresponding Stokes multipliers were studied in [20]. In particular, the functions $S_{j}(x, \lambda)$ are entire in $\lambda$ of order $1 / 2$, and form an FSS of (2.5). Moreover,

$$
\left\langle S_{1}(x, \lambda), S_{2}(x, \lambda)\right\rangle \equiv 1
$$

where $\langle y(x), \tilde{y}(x)\rangle:=y(x) \tilde{y}^{\prime}(x)-y^{\prime}(x) \tilde{y}(x)$ is the Wronskian, furthermore,

$$
\left|S_{j}(x, \lambda)\right| \leq C\left|x^{\mu_{j}}\right| \quad \text { for }|\rho x| \leq 1 .
$$

Here and below, one and the same symbol $C$ denote various positive constants in the estimates. We will call $S_{j}(x, \lambda), j=1,2$, the Bessel-type solutions for (2.5) related to $x=0$. Let $S_{j 1}(x, \lambda), j=1,2,0<x<T$, be the Bessel-type solutions for the equation

$$
-y_{1}^{\prime \prime}(x)+q(T-x) y_{1}(x)=\lambda y_{1}(x)
$$

related to $x=0$. Then the functions $S_{j}^{+}(x, \lambda):=(-1)^{j-1} S_{j 1}(T-x, \lambda), j=1,2$, are solutions of (2.5). They are called the Bessel-type solutions for (2.5) related to $x=T$. Clearly,

$$
\begin{gathered}
\left\langle S_{1}^{+}(x, \lambda), S_{2}^{+}(x, \lambda)\right\rangle \equiv 1, \\
\left|S_{j}^{+}(x, \lambda)\right| \leq C\left|(T-x)^{\mu_{j}^{+}}\right| \quad \text { for }|\rho(T-x)| \leq 1,
\end{gathered}
$$

where $\mu_{j}^{+}=(-1)^{j} \gamma+1 / 2$. 
We introduce the linear forms

$$
\sigma_{k}(y):=(-1)^{k-1}\left\langle y(x), S_{3-k}(x, \lambda)\right\rangle_{\mid x=0}, \quad \sigma_{k}^{+}(y):=(-1)^{k-1}\left\langle y(x), S_{3-k}^{+}(x, \lambda)\right\rangle_{\mid x=T}, \quad k=1,2 .
$$

It follows from (2.10) and (2.13) that

$$
\sigma_{k}\left(S_{j}\right)=\sigma_{k}^{+}\left(S_{j}^{+}\right)=\delta_{j k}, \quad j, k=1,2,
$$

where $\delta_{j k}$ is the Kronecker symbol.

Obviously, the Cauchy-type problem for (2.5) with the initial conditions $\sigma_{k}(y)=c_{k}$, $k=1,2$, has a unique solution, namely, $y(x)=c_{1} S_{1}(x, \lambda)+c_{2} S_{2}(x, \lambda)$. Similarly, the Cauchytype problem for (2.5) with the initial conditions $\sigma_{k}^{+}(y)=c_{k}, k=1,2$, has a unique solution, namely, $y(x)=c_{1} S_{1}^{+}(x, \lambda)+c_{2} S_{2}^{+}(x, \lambda)$.

Remark 2.1. For the classical Sturm-Liouville equation, one has $v=\gamma=1 / 2$ (i.e., $\omega=$ $\left.\omega_{1}=0\right)$; hence $\sigma_{k}(y)=y^{(k-1)}(0), \sigma_{k}^{+}(y)=y^{(k-1)}(T), k=1,2$.

If $\operatorname{Re} \nu, \operatorname{Re} \gamma \in(0,1)$, we have the limit-circle case at both endpoints of the interval $(0, T)$. This case was treated in $[4]$; here we study the general case.

The linear forms $\sigma_{k}(y)$ and $\sigma_{k}^{+}(y)$ allow one to introduce singular two-point boundary conditions of the following general form for (2.5):

$$
a_{k 1} \sigma_{1}(y)+a_{k 2} \sigma_{2}(y)+a_{k 1}^{+} \sigma_{1}^{+}(y)+a_{k 2}^{+} \sigma_{2}^{+}(y)=0, \quad k=1,2,
$$

where

$$
\operatorname{rank}\left[\begin{array}{llll}
a_{11} & a_{12} & a_{11}^{+} & a_{12}^{+} \\
a_{21} & a_{22} & a_{21}^{+} & a_{22}^{+}
\end{array}\right]=2 .
$$

It is natural and convenient to normalize the boundary conditions (2.17) (compare the similar procedure in [13] for classical boundary value problems without singularities). This normalization procedure gives us 3 classes of the boundary conditions (2.17).

Case 1. Let $\operatorname{rank}\left[a_{k 2}, a_{k 2}^{+}\right]_{k=1,2}=2$. Then solving (2.17) with respect to $\sigma_{2}(y)$ and $\sigma_{2}^{+}(y)$, we arrive at the equivalent boundary conditions of the form

$$
\begin{aligned}
& U_{1}(y):=\sigma_{2}(y)+a_{1} \sigma_{1}(y)+a_{1}^{+} \sigma_{1}^{+}(y)=0, \\
& U_{2}(y):=\sigma_{2}^{+}(y)+a_{2} \sigma_{1}(y)+a_{2}^{+} \sigma_{1}^{+}(y)=0 .
\end{aligned}
$$

Case 2. Let $\operatorname{rank}\left[a_{k 2}, a_{k 2}^{+}\right]_{k=1,2}=1$. Then the boundary conditions (2.17) can be reduced to the form

$a_{11} \sigma_{1}(y)+a_{12} \sigma_{2}(y)+a_{11}^{+} \sigma_{1}^{+}(y)+a_{12}^{+} \sigma_{2}^{+}(y)=a_{01} \sigma_{1}(y)+a_{01}^{+} \sigma_{1}^{+}(y)=0, \quad\left|a_{12}\right|+\left|a_{11}^{+}\right|>0$.

Case 3. Let $\operatorname{rank}\left[a_{k 2}, a_{k 2}^{+}\right]_{k=1,2}=0$, that is, $a_{k 2}=a_{k 2}^{+}=0, k=1,2$. Then (2.17) can be reduced to the separated boundary conditions of the form $\sigma_{1}(y)=\sigma_{1}^{+}(y)=0$.

For definiteness, we will consider below the boundary conditions of the form (2.19). All other cases are treated analogously. 
Remark 2.2. Similarly, one can introduce singular boundary conditions also for (1.1). Denote

$$
\{z(t), \tilde{z}(t)\}:=p_{2}(t)\left(z(t) \frac{d \tilde{z}(t)}{d t}-\tilde{z}(t) \frac{d z(t)}{d t}\right) .
$$

Then

$$
\{z(t), \tilde{z}(t)\}=\langle y(x), \tilde{y}(x)\rangle,
$$

where $y(x)=\left(p_{0}(t) p_{2}(t)\right)^{1 / 4} z(t), \tilde{y}(x)=\left(p_{0}(t) p_{2}(t)\right)^{1 / 4} \tilde{z}(t), x=\int_{a}^{t} R(\xi) d \xi$. Moreover, if $z(t)$ and $\tilde{z}(t)$ are solutions of (1.1), then the expression $\{z(t), \tilde{z}(t)\}$ does not depend on $t$.

Let

$$
\begin{gathered}
s_{j}(t, \lambda):=\left(p_{0}(t) p_{2}(t)\right)^{1 / 4} S_{j}(x, \lambda), \quad s_{j}^{+}(t, \lambda):=\left(p_{0}(t) p_{2}(t)\right)^{1 / 4} S_{j}^{+}(x, \lambda), \quad x=\int_{a}^{t} R(\xi) d \xi, \\
\tau_{k}(z):=(-1)^{k-1}\left\{z(t), s_{3-k}(t, \lambda)\right\}_{\mid t=a}, \quad \tau_{k}^{+}(z):=(-1)^{k-1}\left\{z(t), s_{3-k}^{+}(t, \lambda)\right\}_{\mid t=b}, \quad k=1,2 .
\end{gathered}
$$

Then the functions $s_{j}(t, \lambda)$ and $s_{j}^{+}(t, \lambda)$ are solutions of $(1.1)$ and $\tau_{k}(z)=\sigma_{k}(y), \tau_{k}^{+}(z)=$ $\sigma_{k}^{+}(y), k=1,2$. Hence, the linear forms $\tau_{k}(z)$ and $\tau_{k}^{+}(z)$ allow one to introduce singular two-point boundary conditions of the general form for (1.1):

$$
a_{k 1} \tau_{1}(z)+a_{k 2} \tau_{2}(z)+a_{k 1}^{+} \tau_{1}^{+}(z)+a_{k 2}^{+} \tau_{2}^{+}(z)=0, \quad k=1,2
$$

\section{Asymptotics of the spectrum}

We consider the boundary value problem $L$ for (2.5) with the boundary conditions (2.19). The main result of this section is the following theorem.

Theorem 3.1. The boundary value problem L has a countable set of eigenvalues $\left\{\lambda_{n}\right\}_{n \geq 0}$. For $n \rightarrow \infty$,

$$
\rho_{n}:=\sqrt{\lambda_{n}}=\frac{\pi}{T}\left(n+p+\frac{\mu_{1}+\mu_{1}^{+}}{2}+O\left(\frac{1}{n^{\beta}}\right)\right),
$$

where $\beta:=\min (1,2 \operatorname{Re} v, 2 \operatorname{Re} \gamma)$, and $p \in \mathbb{Z}$ does not depend on $q_{0}(x), a_{k}, a_{k}^{+}$, and depends only on $\nu, \gamma$.

Proof. Since the functions $S_{j}(x, \lambda), j=1,2$, form an FSS for (2.5), one has

$$
S_{k}^{+}(x, \lambda)=\alpha_{k 1}(\lambda) S_{1}(x, \lambda)+\alpha_{k 2}(\lambda) S_{2}(x, \lambda), \quad 0<x<T, k=1,2 .
$$

Using (2.10), (2.13), and (2.16), we calculate

$$
\begin{gathered}
\alpha_{11}(\lambda)=\sigma_{1}\left(S_{1}^{+}\right)=\sigma_{2}^{+}\left(S_{2}\right), \quad \alpha_{12}(\lambda)=\sigma_{2}\left(S_{1}^{+}\right)=-\sigma_{2}^{+}\left(S_{1}\right), \\
\alpha_{21}(\lambda)=\sigma_{1}\left(S_{2}^{+}\right)=-\sigma_{1}^{+}\left(S_{2}\right), \quad \alpha_{22}(\lambda)=\sigma_{2}\left(S_{2}^{+}\right)=\sigma_{1}^{+}\left(S_{1}\right), \\
\operatorname{det}\left[\alpha_{k j}(\lambda)\right]_{k, j=1,2}=\operatorname{det}\left[\sigma_{k}\left(S_{j}^{+}\right)\right]_{k, j=1,2}=\operatorname{det}\left[\sigma_{k}^{+}\left(S_{j}\right)\right]_{k, j=1,2}=1 .
\end{gathered}
$$


Denote $Z_{k_{0}}=\left\{\rho: \arg \rho \in\left(k_{0} \pi / 2,\left(k_{0}+1\right) \pi / 2\right)\right\}, k_{0}=-1,0$. In each sector $Z_{k_{0}}$, the roots $R_{k}, k=1,2$ of the equation $\xi^{2}+1=0$ can be numbered in such a way that $\operatorname{Re}\left(\rho R_{1}\right)<$ $\operatorname{Re}\left(\rho R_{2}\right), \rho \in Z_{k_{0}}$. Clearly, $R_{k}=(-1)^{k-1} i$ for $Z_{0}$, and $R_{k}=(-1)^{k} i$ for $Z_{-1}$. In [20], for each sector $Z_{k_{0}}$, a special fundamental system of solutions $\left\{y_{k}(x, \rho)\right\}_{k=1,2}, 0<x<T, \rho \in Z_{k_{0}}$ of the differential equation (2.5) has been constructed, having the following properties.

(1) For each $x \in(0, T)$, the functions $y_{k}^{(m)}(x, \rho), m=0,1$, are holomorphic with respect to $\rho$ for $\rho \in Z_{k_{0}},|\rho| \geq \rho_{*}$, are continuous for $\rho \in \overline{Z_{k_{0}}},|\rho| \geq \rho_{*}$, and

$$
y_{k}^{(m)}(x, \rho)=\left(\rho R_{k}\right)^{m} \exp \left(\rho R_{k} x\right)[1]_{0}, \quad x \in(0, T), \rho \in \overline{Z_{k_{0}}},|\rho x| \geq 1,|\rho(T-x)| \geq 1 \text {, }
$$

where $[1]_{0}=1+O\left((\rho x)^{-\beta}\right)+O\left((\rho(T-x))^{-\beta}\right)$, that is, $f(x, \rho)=[1]_{0}$ means that $|f(x, \rho)-1| \leq C\left(|\rho x|^{-\beta}+|\rho(T-x)|^{-\beta}\right)$.

(2) The relation

$$
S_{j}(x, \lambda)=\sum_{k=1}^{2} d_{j k}(\rho) y_{k}(x, \rho), \quad 0<x<T,
$$

holds, where

$$
d_{j 1}(\rho)=d_{j} \exp \left(-i \pi \mu_{j}\right) \rho^{-\mu_{j}}[1], \quad d_{j 2}(\rho)=d_{j} \rho^{-\mu_{j}}[1], \quad d_{1} d_{2}=-(4 i \sin \pi \nu)^{-1} .
$$

Here and below, [1] $=1+O\left(\rho^{-\beta}\right)$. We will call $y_{k}(x, \rho), k=1,2$, the Birkhoff-type solutions for (2.5) related to $x=0$.

Let $y_{k 1}(x, \rho), k=1,2$, be the Birkhoff-type solutions for (2.12) related to $x=0$. Then the functions $y_{k}^{+}(x, \rho):=y_{k 1}(T-x, \rho)$ are solutions of $(2.5)$, and

$$
\begin{gathered}
\frac{d^{m}}{d x^{m}} y_{k}^{+}(x, \rho)=\left(\rho R_{k}\right)^{m} \exp \left(\rho R_{k}(T-x)\right)[1]_{0}, \\
x \in(0, T), \rho \in \overline{Z_{k_{0}}},|\rho x| \geq 1,|\rho(T-x)| \geq 1, \\
S_{j}^{+}(x, \lambda)=(-1)^{j-1} \sum_{k=1}^{2} d_{j k}^{+}(\rho) y_{k}^{+}(x, \rho), \quad 0<x<T,
\end{gathered}
$$

where

$$
d_{j 1}^{+}(\rho)=d_{j}^{+} \exp \left(-i \pi \mu_{j}^{+}\right) \rho^{-\mu_{j}^{+}}[1], \quad d_{j 2}^{+}(\rho)=d_{j}^{+} \rho^{-\mu_{j}^{+}}[1], \quad d_{1}^{+} d_{2}^{+}=-(4 i \sin \pi \gamma)^{-1} .
$$

We will call $y_{k}^{+}(x, \rho), k=1,2$, the Birkhoff-type solutions for (2.5) related to $x=T$. 
It follows from (3.6) and (3.9) that

$$
\begin{gathered}
y_{k}(x, \rho)=\sum_{j=1}^{2} b_{k j}(\rho) S_{j}(x, \lambda), \quad 0<x<T, \\
y_{k}^{+}(x, \rho)=\sum_{j=1}^{2} b_{k j}^{+}(\rho)(-1)^{j-1} S_{j}^{+}(x, \lambda), \quad 0<x<T,
\end{gathered}
$$

where

$$
b_{k j}(\rho)=\beta_{k j} \rho^{\mu_{j}}[1], \quad b_{k j}^{+}(\rho)=\beta_{k j}^{+} \rho^{\mu_{j}^{+}}[1], \quad|\rho| \longrightarrow \infty,
$$

and $\beta_{k j}, \beta_{k j}^{+}$are complex numbers. It follows from (3.5), (3.6), (3.8), and (3.9) that for $|\rho| \rightarrow \infty,|\rho| x \geq 1,|\rho|(T-x) \geq 1$, the following asymptotic formulae are valid:

$$
\begin{gathered}
S_{j}^{(m)}(x, \lambda)=d_{j} \rho^{-\mu_{j}}\left((-i \rho)^{m} \exp (-i \rho x)[1]_{0}+(i \rho)^{m} \exp \left(-i \pi \mu_{j}\right) \exp (i \rho x)[1]_{0}\right), \\
S_{j}^{+(m)}(x, \lambda)=(-1)^{j-1} d_{j}^{+} \rho^{-\mu_{j}^{+}}\left((i \rho)^{m} \exp (-i \rho(T-x))[1]_{0}\right. \\
\left.+(-i \rho)^{m} \exp \left(-i \pi \mu_{j}^{+}\right) \exp (i \rho(T-x))[1]_{0}\right) .
\end{gathered}
$$

In order to find the asymptotic behavior of $\alpha_{k j}(\lambda)$, we substitute (3.14) into (3.2):

$$
\begin{aligned}
(-1)^{k-1} & d_{k}^{+} \rho^{-\mu_{k}^{+}}\left(\exp (-i \rho(T-x))[1]_{0}+\exp \left(-i \pi \mu_{k}^{+}\right) \exp (i \rho(T-x))[1]_{0}\right) \\
= & \alpha_{k 1}(\lambda) d_{1} \rho^{-\mu_{1}}\left(\exp (-i \rho x)[1]_{0}+\exp \left(-i \pi \mu_{1}\right) \exp (i \rho x)[1]_{0}\right) \\
& +\alpha_{k 2}(\lambda) d_{2} \rho^{-\mu_{2}}\left(\exp (-i \rho x)[1]_{0}+\exp \left(-i \pi \mu_{2}\right) \exp (i \rho x)[1]_{0}\right) .
\end{aligned}
$$

Since $x$ is arbitrary from $(0, T)$, we infer

$\alpha_{k j}(\lambda)=2 i(-1)^{k-j+1} d_{3-j} d_{k}^{+} \rho^{1-\mu_{3-j}-\mu_{k}^{+}}\left(\exp (-i \rho T)[1]-\exp \left(-i \pi\left(\mu_{3-j}+\mu_{k}^{+}\right)\right) \exp (i \rho T)[1]\right)$.

Therefore,

$$
\left|\alpha_{k j}(\lambda)\right| \leq C\left|\rho^{1-\mu_{3-j}-\mu_{k}^{+}}\right| \exp (|\operatorname{Im} \rho| T)
$$

Denote

$$
\Delta(\lambda):=\operatorname{det}\left[U_{k}\left(S_{j}\right)\right]_{k, j=1,2} .
$$

The function $\Delta(\lambda)$ is entire in $\lambda$ of order $1 / 2$, and its zeros $\left\{\lambda_{n}\right\}$ coincide with the eigenvalues of the boundary value problem $L$. The function $\Delta(\lambda)$ is called the characteristic function for $L$. Taking (2.16), (2.19), (3.3), and (3.4) into account, we calculate

$$
\Delta(\lambda)=\alpha_{12}(\lambda)-a_{2}^{+} \alpha_{22}(\lambda)+a_{1} \alpha_{11}(\lambda)+\left(a_{2} a_{1}^{+}-a_{1} a_{2}^{+}\right) \alpha_{21}(\lambda)+a_{1}^{+}-a_{2} .
$$

Substituting (3.16) into (3.19), we obtain the following asymptotic formula for the characteristic function $\Delta(\lambda)$ for $|\rho| \rightarrow \infty$ :

$$
\Delta(\lambda)=2 i d_{1}^{+} d_{1} \rho^{\nu+\gamma}\left(\exp (-i \rho T)[1]-\exp \left(-i \pi\left(\mu_{1}+\mu_{1}^{+}\right)\right) \exp (i \rho T)[1]\right) .
$$


Using (3.20) and Rouche's theorem [2, page 125], we arrive in the usual way (see [13, Chapter 1]) at (3.1).

Fix $\delta>0$. Denote $G_{\delta}:=\left\{\rho:\left|\rho-\rho_{n}\right| \geq \delta, n \geq 0\right\}$. By the well-known method [13], one can get the estimate

$$
|\Delta(\lambda)| \geq C\left|\rho^{\nu+\gamma}\right| \exp (|\operatorname{Im} \rho| T), \quad \rho \in G_{\delta} .
$$

Moreover, in view of (3.2), (3.4), and (3.18), one has

$$
\Delta(\lambda):=\operatorname{det}\left[U_{k}\left(S_{j}^{+}\right)\right]_{k, j=1,2}
$$

\section{The completeness theorem}

In this section, we prove that the system of eaf's of the boundary value problem $L$ is complete in corresponding Banach spaces. At the end of the section, we provide an analogous theorem for boundary value problems for (1.1) with singular boundary conditions.

Let $\alpha, \eta$ be real numbers and let $1 \leq p<\infty$. We consider the Banach spaces $B_{\alpha, \eta, p}=$ $\left\{f(x): f(x) x^{-\alpha}(T-x)^{-\eta} \in \mathscr{L}_{p}(0, T)\right\}$ with the norm $\|f\|_{\alpha, \eta, p}=\left\|f(x) x^{-\alpha}(T-x)^{-\eta}\right\|_{p}$, where $\|\cdot\|_{p}$ is the norm in the space $\mathscr{L}_{p}(0, T)$. It was proved in [22] that

$$
B_{\alpha, \eta, p} \subseteq B_{\beta, \xi, s}, \quad 1 \leq s \leq p<\infty, \beta-\alpha<s^{-1}-p^{-1}, \xi-\eta<s^{-1}-p^{-1},
$$

(here the symbol $\subseteq$ denotes dense embedding [8, page 9]). In particular, it follows from (4.1) that $B_{\alpha, \eta, p} \subseteq \mathscr{L}_{s}(0, T)$ for $1 \leq s \leq p<\infty, \alpha>p^{-1}-s^{-1}, \eta>p^{-1}-s^{-1}$.

THeOREM 4.1. The system of eaf's of the boundary value problem $L$ is complete in the space $B_{\beta, \xi, s}$ for $1 \leq s<\infty, \beta<\theta+1 / s, \xi<\theta_{1}+1 / s$.

Proof. Let $\left\{\psi_{\ell}(x)\right\}_{\ell \geq 0}$ be the system of eaf's of $L$, and let the function $f(x)$ be such that

$$
f(x) x^{\theta}(T-x)^{\theta_{1}} \in \mathscr{L}(0, T), \quad \int_{0}^{T} f(x) \psi_{\ell}(x) d x=0 \quad \text { for } \ell \geq 0 .
$$

Denote

$$
\varphi_{k}(x, \lambda)=U_{k}\left(S_{2}\right) S_{1}(x, \lambda)-U_{k}\left(S_{1}\right) S_{2}(x, \lambda), \quad 0<x<T, k=1,2 .
$$

The functions $\varphi_{k}(x, \lambda)$ are solutions of (2.5), and in view of (3.18),

$$
U_{k}\left(\varphi_{k}\right)=0, \quad k=1,2, \quad U_{1}\left(\varphi_{2}\right)=-U_{2}\left(\varphi_{1}\right)=\Delta(\lambda) .
$$

The functions $\varphi_{k}(x, \lambda), k=1,2$, are entire in $\lambda$ of order $1 / 2$. For $\lambda=\lambda_{n}, n \geq 0$, the functions $\varphi_{k}\left(x, \lambda_{n}\right)$ satisfy the boundary conditions (2.19). Taking (2.10), (3.18), and (4.3) into account, we obtain

$$
\left\langle\varphi_{1}(x, \lambda), \varphi_{2}(x, \lambda)\right\rangle \equiv \Delta(\lambda)
$$

By virtue of (3.22) and (4.4),

$$
\varphi_{k}(x, \lambda)=U_{k}\left(S_{2}^{+}\right) S_{1}^{+}(x, \lambda)-U_{k}\left(S_{1}^{+}\right) S_{2}^{+}(x, \lambda), \quad 0<x<T, k=1,2 .
$$


Denote

$$
F_{k}(\lambda)=\int_{0}^{T} f(x) \varphi_{k}(x, \lambda) d x, \quad Q_{k}(\lambda)=(\Delta(\lambda))^{-1} F_{k}(\lambda), \quad k=1,2 .
$$

It follows from (4.2), (4.4), (4.5), and (4.7) that the functions $Q_{k}(\lambda)$ are entire in $\lambda$, since all its singularities are removable. In order to estimate $\left|Q_{k}(\lambda)\right|$, we need the following auxiliary assertion.

Lemma 4.2. For $\rho \in G_{\delta},|\rho| x \geq 1,|\rho|(T-x) \geq 1$,

$$
\left|(\Delta(\lambda))^{-1} \varphi_{k}(x, \lambda)\right| \leq C|\rho|^{-1 / 2-\varepsilon},
$$

where $\varepsilon:=\min (\operatorname{Re} \nu, \operatorname{Re} \gamma)>0$.

Proof. It follows from (2.10), (3.11), and (3.18) that

$$
\left\langle y_{1}(x, \rho), y_{2}(x, \rho)\right\rangle=\operatorname{det}\left[b_{k j}(\rho)\right]_{k, j=1,2}, \quad \operatorname{det}\left[U_{\xi}\left(y_{k}\right)\right]_{\xi, k=1,2}=\Delta(\lambda) \operatorname{det}\left[b_{k j}(\rho)\right]_{k, j=1,2},
$$

and consequently,

$$
\operatorname{det}\left[U_{\xi}\left(y_{k}\right)\right]_{\xi, k=1,2}=\Delta(\lambda)\left\langle y_{1}(x, \rho), y_{2}(x, \rho)\right\rangle .
$$

In view of (4.4) and (4.10), we get

$$
\varphi_{k}(x, \lambda)=\left(\left\langle y_{1}(x, \rho), y_{2}(x, \rho)\right\rangle\right)^{-1}\left(U_{k}\left(y_{2}\right) y_{1}(x, \rho)-U_{k}\left(y_{1}\right) y_{2}(x, \rho)\right), \quad k=1,2 .
$$

It follows from (2.16), (3.11), and (3.12) that

$$
\sigma_{\xi}\left(y_{k}\right)=b_{k \xi}(\rho), \quad \sigma_{\xi}^{+}\left(y_{k}^{+}\right)=b_{k \xi}^{+}(\rho) .
$$

Since the functions $y_{j}^{+}(x, \rho), j=1,2$, form an FSS for (2.5), one has

$$
y_{k}(x, \rho)=\sum_{j=1}^{2} \Gamma_{k j}(\rho) y_{j}^{+}(x, \rho)
$$

Let for definiteness, $\rho \in \overline{Z_{0}}$, that is, $\arg \rho \in[0, \pi / 2]$. Then

$$
\begin{aligned}
y_{1}(x, \rho)=\exp (i \rho x)[1]_{0}, & y_{2}(x, \rho)=\exp (-i \rho x)[1]_{0}, \\
y_{1}^{+}(x, \rho)=\exp (i \rho(T-x))[1]_{0}, & y_{2}^{+}(x, \rho)=\exp (-i \rho(T-x))[1]_{0},
\end{aligned}
$$

and consequently, for $|\rho| \rightarrow \infty, \rho \in \overline{Z_{0}}$,

$$
\begin{gathered}
\Gamma_{12}(\rho)=\exp (i \rho T)[1], \quad \Gamma_{21}(\rho)=\exp (-i \rho T)[1] \\
\Gamma_{k k}(\rho)=O\left(\rho^{-\beta}\right) \exp (i \rho T), \quad k=1,2
\end{gathered}
$$


It follows from (4.12), (4.13), (4.14), and (4.15) that for $|\rho| \rightarrow \infty, \rho \in \overline{Z_{0}}$,

$$
\sigma_{\xi}^{+}\left(y_{1}\right)=(-1)^{\xi-1} b_{2 \xi}^{+}(\rho) \exp (i \rho T)[1], \quad \sigma_{\xi}^{+}\left(y_{2}\right)=(-1)^{\xi-1} b_{1 \xi}^{+}(\rho) \exp (-i \rho T)[1] .
$$

Substituting (4.12) and (4.16) into (2.19) and taking (3.13) into account, we obtain for $|\rho| \rightarrow \infty, \rho \in \overline{Z_{0}}$,

$$
\begin{gathered}
U_{1}\left(y_{1}\right)=\beta_{12} \rho^{1 / 2+\nu}[1], \quad U_{1}\left(y_{2}\right)=\beta_{22} \rho^{1 / 2+\nu}[1]+a_{1}^{+} \beta_{11}^{+} \rho^{1 / 2-\gamma} \exp (-i \rho T)[1], \\
U_{2}\left(y_{1}\right)=-\beta_{22}^{+} \rho^{1 / 2+\gamma}[1] \exp (i \rho T)[1]+a_{2} \rho^{1 / 2-\gamma}[1] \\
U_{2}\left(y_{2}\right)=-\beta_{12}^{+} \rho^{1 / 2+\gamma}[1] \exp (-i \rho T)[1] .
\end{gathered}
$$

Since $\left\langle y_{1}(x, \rho), y_{2}(x, \rho)\right\rangle=-2 i \rho[1]$ as $|\rho| \rightarrow \infty, \rho \in \overline{Z_{0}}$, it follows from (4.11), (4.14), and (4.17) that for $|\rho| \rightarrow \infty,|\rho| x \geq 1,|\rho|(T-x) \geq 1, \rho \in \overline{Z_{0}}$,

$$
\begin{aligned}
\varphi_{1}(x, \lambda)= & \frac{1}{2 i}\left(\beta_{12} \rho^{\nu-1 / 2} \exp (-i \rho x)[1]_{0}\right. \\
& \left.-\left(\beta_{22} \rho^{\nu-1 / 2}[1]+a_{1}^{+} \beta_{11}^{+} \rho^{-1 / 2-\gamma} \exp (-i \rho T)[1]\right) \exp (i \rho x)[1]_{0}\right) \\
\varphi_{2}(x, \lambda)= & \frac{1}{2 i}\left(\left(-\beta_{22}^{+} \rho^{-1 / 2+\gamma} \exp (i \rho T)[1]+a_{2} \rho^{-1 / 2-\gamma}[1]\right) \exp (-i \rho x)[1]_{0}\right. \\
& \left.+\beta_{12}^{+} \rho^{-1 / 2+\gamma} \exp (-i \rho T) \exp (i \rho x)[1]_{0}\right)
\end{aligned}
$$

In particular, together with (3.21), this yields (4.8) for $\rho \in \overline{Z_{0}}$. For $\rho \in \overline{Z_{-1}}$, the arguments are similar. Lemma 4.2 is proved.

Now we return to the proof of Theorem 4.1. We show that

$$
\left|Q_{k}(\lambda)\right| \leq C|\rho|^{-\varepsilon}, \quad \rho \in G_{\delta} .
$$

For this purpose, we denote $\gamma_{\rho, 0}=\{x \in[0, T]|| \rho \mid x \leq 1\}, \gamma_{\rho, 1}=\{x \in[0, T]|| \rho \mid(T-x) \leq$ $1\}, \gamma_{\rho, 2}=[0, T] \backslash\left(\gamma_{\rho, 0} \cup \gamma_{\rho, 1}\right)$. Then

$$
Q_{k}(\lambda)=Q_{k 0}(\lambda)+Q_{k 1}(\lambda)+Q_{k 2}(\lambda), \quad Q_{k j}(\lambda):=(\Delta(\lambda))^{-1} \int_{\gamma_{\rho, j}} f(x) \varphi_{k}(x, \lambda) d x .
$$

Since $f(x)=f_{0}(x) x^{\nu-1 / 2}(T-x)^{\gamma-1 / 2}, f_{0}(x) \in \mathscr{L}(0, T)$, we have by virtue of (4.8),

$$
\begin{aligned}
& \left|Q_{k 2}(\lambda)\right| \\
& \quad \leq C|\rho|^{-1 / 2-\varepsilon} \int_{\gamma_{\rho, 2}}\left|f_{0}(x) x^{\nu-1 / 2}(T-x)^{\gamma-1 / 2}\right| d x \\
& \quad \leq C|\rho|^{-\varepsilon}\left(\int_{1 /|\rho|}^{T / 2}\left|f_{0}(x) x^{\nu}(T-x)^{\gamma-1 / 2}\right| d x+\int_{T / 2}^{T-1 /|\rho|}\left|f_{0}(x) x^{\nu-1 / 2}(T-x)^{\gamma}\right| d x\right) \\
& \quad \leq C|\rho|^{-\varepsilon} \int_{0}^{T}\left|f_{0}(x)\right| d x,
\end{aligned}
$$


hence

$$
\left|Q_{k 2}(\lambda)\right| \leq C|\rho|^{-\varepsilon}, \quad \rho \in G_{\delta}
$$

Furthermore, using (2.16), (2.19), (3.3), and (3.17), we obtain

$$
\begin{aligned}
\left|U_{1}\left(S_{1}\right)\right| \leq C\left(1+\left|\rho^{\nu-\gamma}\right| \exp (|\operatorname{Im} \rho| T)\right), & \left|U_{2}\left(S_{1}\right)\right| \leq C\left|\rho^{\nu+\gamma}\right| \exp (|\operatorname{Im} \rho| T), \\
\left|U_{1}\left(S_{2}\right)\right| \leq C\left(1+\left|\rho^{-\gamma-\gamma}\right| \exp (|\operatorname{Im} \rho| T)\right), & \left|U_{2}\left(S_{2}\right)\right| \leq C\left|\rho^{-\gamma+\gamma}\right| \exp (|\operatorname{Im} \rho| T) .
\end{aligned}
$$

It follows from (2.11), (3.21), (4.3), (4.20), and (4.23) that

$$
\begin{aligned}
\left|Q_{10}(\lambda)\right| \leq & C\left|\rho^{-\nu-\gamma}\right| \exp (-|\operatorname{Im} \rho| T) \\
& \times \int_{0}^{1 /|\rho|}\left|f_{0}(x) x^{\nu-1 / 2}\right|\left(\left|x^{-\nu+1 / 2}\right|\left(1+\left|\rho^{-\nu-\gamma}\right| \exp (|\operatorname{Im} \rho| T)\right)\right. \\
& \left.\quad+\left|x^{\nu+1 / 2}\right|\left(1+\left|\rho^{\nu-\gamma}\right| \exp (|\operatorname{Im} \rho| T)\right)\right) d x \\
\leq & C\left|\rho^{-\nu-\gamma}\right|\left(\int_{0}^{1 /|\rho|}\left|f_{0}(x)\right| d x+\left(1+\left|\rho^{\nu-\gamma}\right|\right) \int_{0}^{1 /|\rho|}\left|f_{0}(x) x^{2 \gamma}\right| d x\right) \\
\leq & C|\rho|^{-2 \varepsilon}, \quad \rho \in G_{\delta}, \\
\left|Q_{20}(\lambda)\right| \leq & C\left|\rho^{-\nu-\gamma}\right| \int_{0}^{1 /|\rho|}\left|f_{0}(x) x^{\nu-1 / 2}\right|\left(\left|x^{-\nu+1 / 2} \rho^{-\nu+\gamma}\right|+\left|x^{\nu+1 / 2} \rho^{\nu+\gamma}\right|\right) d x \\
\leq & C\left|\rho^{-2 \gamma}\right| \int_{0}^{1 /|\rho|}\left|f_{0}(x)\right| d x+C \int_{0}^{1 /|\rho|}\left|f_{0}(x) x^{2 \nu}\right| d x \\
\leq & C|\rho|^{-2 \varepsilon}, \quad \rho \in G_{\delta} .
\end{aligned}
$$

Thus,

$$
\left|Q_{k 0}(\lambda)\right| \leq C|\rho|^{-2 \varepsilon}, \quad \rho \in G_{\delta}
$$

Similarly, using (2.14), (3.21), and (4.6), one obtains

$$
\left|Q_{k 1}(\lambda)\right| \leq C|\rho|^{-2 \varepsilon}, \quad \rho \in G_{\delta}
$$

Together with (4.22), this yields (4.19).

Since the functions $Q_{k}(\lambda)$ are entire in $\lambda$, it follows from (4.19) and Liouville's theorem that $Q_{k}(\lambda) \equiv 0, k=1,2$, and consequently,

$$
F_{k}(\lambda):=\int_{0}^{T} f(x) \varphi_{k}(x, \lambda) d x \equiv 0, \quad k=1,2 .
$$

Denote

$$
F_{j 0}(\lambda):=\int_{0}^{T} f(x) S_{j}(x, \lambda) d x, \quad j=1,2 .
$$


By virtue of (4.3) and (4.27),

$$
U_{k}\left(S_{2}\right) F_{10}(\lambda)-U_{k}\left(S_{1}\right) F_{20}(\lambda) \equiv 0, \quad k=1,2 .
$$

According to (3.18), the determinant of this linear algebraic system is equal to $\Delta(\lambda)$. Solving this system we get $F_{10}(\lambda)=F_{20}(\lambda) \equiv 0$, that is,

$$
\int_{0}^{T} f(x) S_{j}(x, \lambda) d x \equiv 0, \quad j=1,2 .
$$

Now we consider the boundary value problem $L_{0}$ for (2.5) with the boundary conditions $\sigma_{1}(y)=\sigma_{1}^{+}(y)=0$. The eigenvalues $\left\{\lambda_{n}^{0}\right\}_{n \geq 0}$ of $L_{0}$ coincide with the zeros of the characteristic function

$$
\Delta_{0}(\lambda):=\sigma_{1}^{+}\left(S_{2}\right)=\left\langle S_{2}(x, \lambda), S_{2}^{+}(x, \lambda)\right\rangle
$$

According to (3.3) and (4.31), $\Delta_{0}(\lambda)=-\alpha_{21}(\lambda)$. Using (3.16), one can get

$$
\begin{gathered}
\rho_{n}^{0}:=\sqrt{\lambda_{n}^{0}}=\frac{\pi}{T}\left(n+p_{0}+\frac{\mu_{2}+\mu_{2}^{+}}{2}+O\left(\frac{1}{n^{\beta}}\right)\right), \quad n \longrightarrow \infty, \\
\left|\Delta_{0}(\lambda)\right| \geq C\left|\rho^{-\nu-\gamma}\right| \exp (|\operatorname{Im} \rho| T), \quad \rho \in G_{\delta}^{0},
\end{gathered}
$$

where $p_{0} \in \mathbb{Z}, G_{\delta}:=\left\{\rho:\left|\rho-\rho_{n}^{0}\right| \geq \delta, n \geq 0\right\}$.

We consider the function

$$
y(x, \lambda)=\left(\Delta_{0}(\lambda)\right)^{-1}\left(S_{2}^{+}(x, \lambda) \int_{0}^{x} f(t) S_{2}(t, \lambda) d t+S_{2}(x, \lambda) \int_{x}^{T} f(t) S_{2}^{+}(t, \lambda) d t\right) .
$$

It is easy to check that

$$
y^{\prime \prime}(x, \lambda)-q(x) y(x, \lambda)+\lambda y(x, \lambda)=f(x), \quad 0<x<T .
$$

Fix $x \in(0, T)$, and let $|\rho| x \geq 1,|\rho|(T-x) \geq 1$. Then, according to (3.14),

$$
\left|S_{2}(x, \lambda)\right| \leq C\left|\rho^{-\nu-1 / 2}\right| \exp (|\operatorname{Im} \rho| x), \quad\left|S_{2}^{+}(x, \lambda)\right| \leq C\left|\rho^{-\gamma-1 / 2}\right| \exp (|\operatorname{Im} \rho|(T-x)) .
$$

Using (2.11) and (4.35), we calculate

$$
\begin{array}{r}
\int_{0}^{1 /|\rho|}\left|f(t) S_{2}(t, \lambda)\right| d t \leq C \int_{0}^{1 /|\rho|}\left|f_{0}(t) t^{2 \nu}(T-t)^{-1 / 2+\gamma}\right| d t \leq C\left|\rho^{-2 \nu}\right| \\
\int_{1 /|\rho|}^{x}\left|f(t) S_{2}(t, \lambda)\right| d t \leq C\left|\rho^{-\nu-1 / 2}\right| \int_{1 /|\rho|}^{x}\left|f_{0}(t) t^{\nu-1 / 2}(T-t)^{-1 / 2+\gamma}\right| \exp (|\operatorname{Im} \rho| t) d t \\
\leq C\left|\rho^{-\nu}\right| \exp (|\operatorname{Im} \rho| x) \int_{1 /|\rho|}^{x}\left|f_{0}(t) t^{\nu}\right| d t \leq C\left|\rho^{-\nu}\right| \exp (|\operatorname{Im} \rho| x)
\end{array}
$$


Analogously, using (2.14) and (4.35), we get

$$
\begin{gathered}
\int_{T-1 /|\rho|}^{T}\left|f(t) S_{2}^{+}(t, \lambda)\right| d t \leq C\left|\rho^{-2 \gamma}\right|, \\
\int_{x}^{T-1 /|\rho|}\left|f(t) S_{2}^{+}(t, \lambda)\right| d t \leq C\left|\rho^{-\gamma}\right| \exp (|\operatorname{Im} \rho|(T-x)) .
\end{gathered}
$$

Substituting (4.32), (4.35), (4.36), and (4.37) into (4.33), we obtain

$$
|y(x, \lambda)| \leq C|\rho|^{-1 / 2}, \quad \rho \in G_{\delta},|\rho| x \geq 1,|\rho|(T-x) \geq 1
$$

Furthermore, using (3.2) and (4.33), we calculate

$$
\begin{aligned}
y(x, \lambda)= & -\left(S_{1}(x, \lambda) \int_{0}^{x} f(t) S_{2}(t, \lambda) d t+S_{2}(x, \lambda) \int_{x}^{T} f(t) S_{1}(t, \lambda) d t\right) \\
& -\frac{\alpha_{22}(\lambda)}{\alpha_{21}(\lambda)} S_{2}(x, \lambda) \int_{0}^{T} f(t) S_{2}(t, \lambda) d t .
\end{aligned}
$$

By virtue of (4.30), the last integral is identically zero, and consequently, the function $y(x, \lambda)$ is entire in $\lambda$ for each fixed $x \in(0, T)$. Together with (4.38), this yields $y(x, \lambda) \equiv 0$. In view of (4.35), we conclude that $f(x)=0$ a.e. on $(0, T)$.

Thus, we have proved that for each $p(1 \leq p<\infty)$, the system of eaf's of $L$ is complete in $B_{\theta, \theta_{1}, p}$. Since $\beta<\theta+1 / s, \xi<\theta_{1}+1 / s$, we have $\beta<\theta+1 / s-1 / p, \xi<\theta_{1}+1 / s-1 / p$, for sufficiently large $p$, and according to (4.1), $B_{\theta, \theta_{1}, p} \subseteq B_{\beta, \xi, s}$. Consequently, the system of eaf's of $L$ is complete in $B_{\beta, \xi, s}$ for $1 \leq s<\infty, \beta<\theta+1 / s, \xi<\theta_{1}+1 / s$. Theorem 4.1 is proved.

Corollary 4.3. The system of eaf's of $L$ is complete in $\mathscr{L}_{s}(0, T)$ for $1 / s>\max (\operatorname{Re} v-$ $1 / 2, \operatorname{Re} \gamma-1 / 2)$.

We consider the boundary value problem $Q$ for (1.1) with the boundary conditions

$$
\begin{aligned}
& \tau_{2}(z)+a_{1} \tau_{1}(z)+a_{1}^{+} \tau_{1}^{+}(z)=0, \\
& \tau_{2}^{+}(z)+a_{2} \tau_{1}(z)+a_{2}^{+} \tau_{1}^{+}(z)=0 .
\end{aligned}
$$

The eigenvalues of $Q$ coincide with the eigenvalues of $L$, hence Theorem 3.1 remains true also for $Q$. Denote

$$
w=\frac{\left(s_{0}+2\right) \theta}{2}-\frac{s_{00}+s_{20}}{4}, \quad w_{1}=\frac{\left(s_{1}+2\right) \theta_{1}}{2}-\frac{s_{01}+s_{21}}{4} .
$$

The following theorem is an obvious corollary of Theorem 4.1.

THeOREM 4.4. The system of eaf's of the boundary value problem $Q$ is complete in the space $B_{\beta, \xi, s}$ for $1 \leq s<\infty, \beta<w+1 / s, \xi<w_{1}+1 / s$. In particular, the system of eaf's of $Q$ is complete in $\mathscr{L}_{s}(0, T)$ for $1 / s>\max \left(-w,-w_{1}\right)$. 


\section{References}

[1] F. V. Atkinson, W. N. Everitt, and A. Zettl, Regularization of a Sturm-Liouville problem with an interior singularity using quasiderivatives, Differential Integral Equations 1 (1988), no. 2, 213-221.

[2] J. B. Conway, Functions of One Complex Variable, 2nd ed., vol. I, Springer, New York, 1995.

[3] W. Eberhard, G. Freiling, and K. Wilcken-Stoeber, Indefinite eigenvalue problems with several singular points and turning points, Math. Nachr. 229 (2001), 51-57.

[4] W. Eberhard, G. Freiling, and A. Zettl, Sturm-Liouville problems with singular non-self-adjoint boundary conditions, to appear in Math. Nachr.

[5] G. Freiling, V. S. Rykhlov, and V. A. Yurko, Spectral analysis for an indefinite singular SturmLiouville problem, Appl. Anal. 81 (2002), no. 6, 1283-1305.

[6] G. Freiling and V. A. Yurko, Expansion theorems for boundary value problems with a singularity, Analysis (Munich) 18 (1998), no. 1, 25-39.

[7] M. G. Gasymov, Determination of a Sturm-Liouville equation with a singularity by two spectra, Dokl. Akad. Nauk SSSR 161 (1965), 274-276 (Russian), English translation in Soviet Math. Dokl. 6 (1965), 396-399.

[8] S. G. Krein, Y. I. Petunin, and E. M. Semenov, Interpolation of Linear Operators, Nauka, Moscow, 1978, English translation in American Mathematical Society, Rhode Island 1982.

[9] R. E. Langer, The boundary problem associated with a differential equation in which the coefficient of the parameter changes sign, Trans. Amer. Math. Soc. 31 (1929), no. 1, 1-24.

[10] J. Locker, Spectral Theory of Non-Self-Adjoint Two-Point Differential Operators, Mathematical Surveys and Monographs, vol. 73, American Mathematical Society, Rhode Island, 2000.

[11] J. McHugh, An historical survey of ordinary linear differential equations with a large parameter and turning points, Arch. Hist. Exact Sci. 7 (1970), 277-324.

[12] R. Mennicken and M. Möller, Non-Self-Adjoint Boundary Eigenvalue Problems, North-Holland Mathematics Studies, vol. 192, North-Holland, Amsterdam, 2003.

[13] M. A. Naimark, Linear Differential Operators, 2nd ed., Nauka, Moscow, 1969, English translation of 1st ed., Parts I, II, Ungar, New York, 967, 1968.

[14] A. M. Savchuk and A. A. Shkalikov, Sturm-Liouville operators with singular potentials, Math. Notes 66 (1999), no. 6, 741-753.

[15] M. H. Stone, Expansion in Bessel functions, Ann. of Math. (2) 28 (1927), no. 1-4, 271-290.

[16] E. C. Titchmarsh, Eigenfunction Expansions Associated with Second-Order Differential Equations, Clarendon Press, Oxford, 1946.

[17] C. Tretter, On ג-Nonlinear Boundary Eigenvalue Problems, Mathematical Research, vol. 71, Akademie-Verlag, Berlin, 1993.

[18] W. Wasow, Linear Turning Point Theory, Applied Mathematical Sciences, vol. 54, Springer, New York, 1985.

[19] S. Yakubov, Completeness of Root Functions of Regular Differential Operators, Pitman Monographs and Surveys in Pure and Applied Mathematics, vol. 71, Longman Scientific \& Technical, Harlow, 1994, copublished in the United States with John Wiley \& Sons, New York.

[20] V. A. Yurko, An inverse problem for differential equations with a singularity, Differ. Uravn. 28 (1992), no. 8, 1355-1362 (Russian), English translation in Differ. Equ. 28 (1992), no. 8, $1100-1107$.

[21] On higher-order differential operators with a regular singularity, Mat. Sb. 186 (1995), no. 6, 133-160 (Russian), English translation in Sb. Math. 186 (1995), no. 6, 901-928.

[22] _ On the reconstruction of Sturm-Liouville differential operators with singularities inside the interval, Mat. Zametki 64 (1998), no. 1, 143-156 (Russian), English translation in Math. Notes, 64 (1998), no. 1, 121-132. 
[23] A. Zettl, Sturm-Liouville problems, Spectral Theory and Computational Methods of SturmLiouville Problems (Knoxville, Tenn, 1996), Lecture Notes in Pure and Appl. Math., vol. 191, Marcel Dekker, New York, 1997, pp. 1-104.

[24] L. A. Zhornitskaya and V. S. Serov, Inverse eigenvalue problems for a singular Sturm-Liouville operator on (0,1), Inverse Problems 10 (1994), no. 4, 975-987.

G. Freiling: Fachbereich Mathematik, Universität Duisburg-Essen, 47048 Duisburg, Germany E-mail address: freiling@math.uni-duisburg.de

V. Yurko: Department of Mathematics and Mechanics, Saratov State University, Astrakhanskaya 83, Saratov 410026, Russia

E-mail address: yurkova@info.sgu.ru 


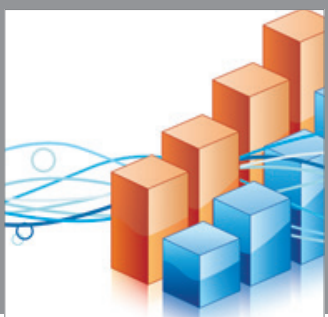

Advances in

Operations Research

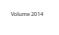

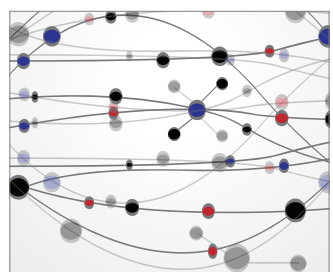

\section{The Scientific} World Journal
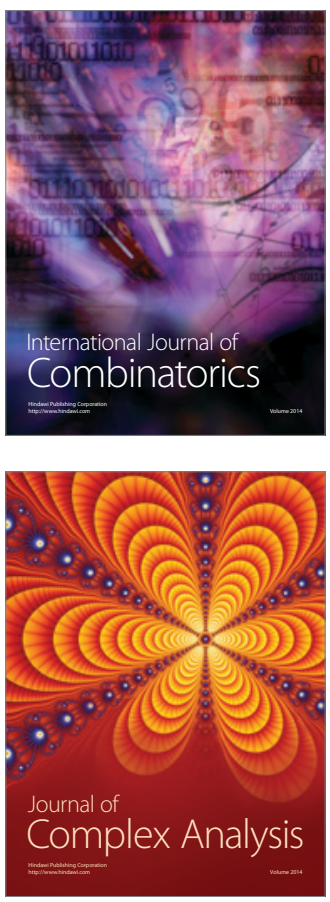

International Journal of

Mathematics and

Mathematical

Sciences
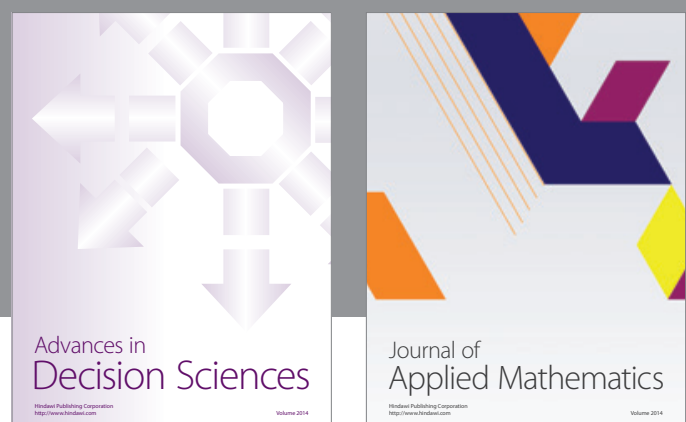

Journal of

Applied Mathematics
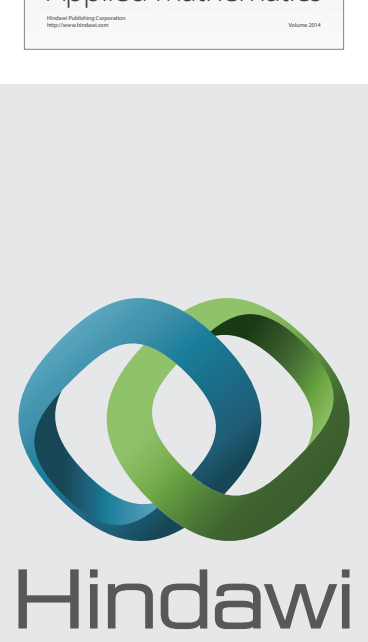

Submit your manuscripts at http://www.hindawi.com
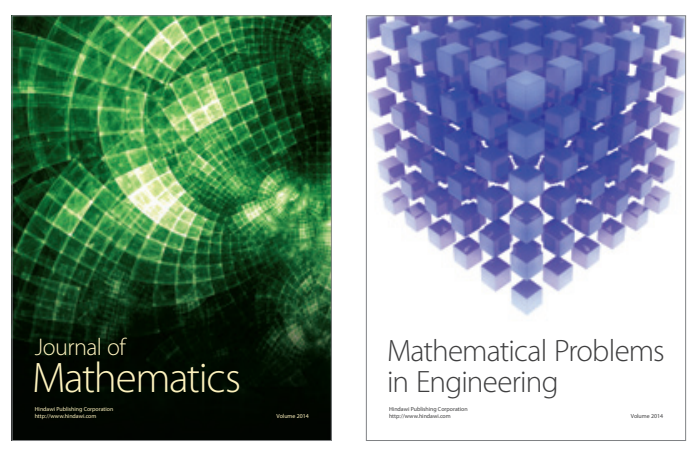

Mathematical Problems in Engineering
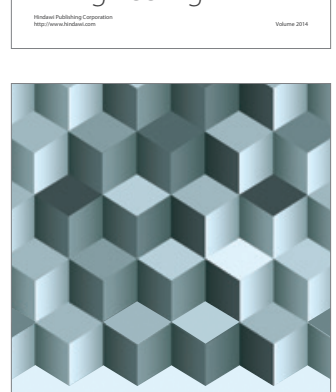

Journal of

Function Spaces
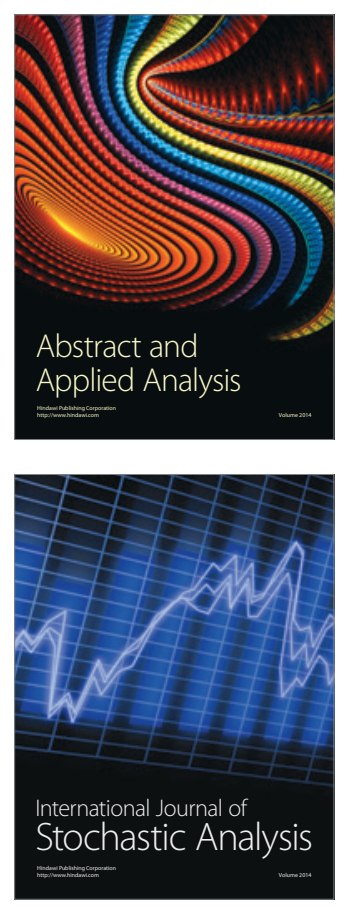

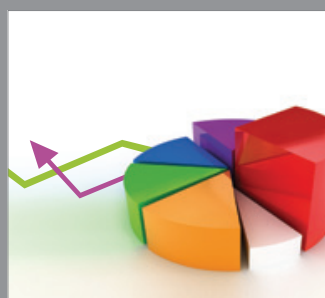

ournal of

Probability and Statistics

Promensencen
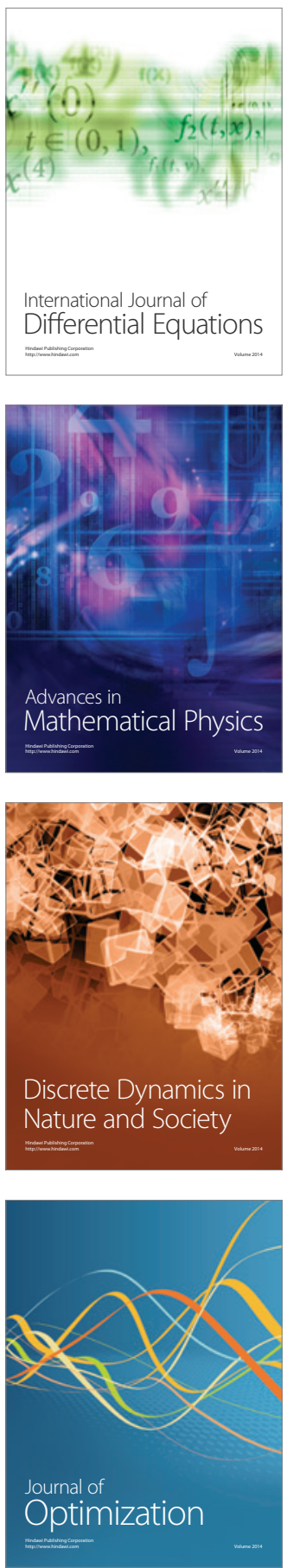